\title{
Whole genome sequencing of SARS-CoV-2 links wastewater RNA to individual cases in catchments.
}

\begin{abstract}
Avram Levy ${ }^{1,5 *}$, Jake Gazeley ${ }^{1}$, Terence Lee ${ }^{1}$, Andrew Jardine², Cameron Gordon ${ }^{3}$, Natalie Cooper $^{1}$, Richard Theobald ${ }^{4}$, Clare Huppatz ${ }^{2}$, Sandra Sjollema ${ }^{1}$, Meredith Hodge ${ }^{1}$ and David Speers $^{1,6}$
\end{abstract}

${ }^{1}$ Department of Microbiology, PathWest Laboratory Medicine WA, Queen Elizabeth II Medical Centre, Nedlands, Western Australia, Australia.

${ }^{2}$ Public Health Emergency Operations Centre, Health Department of Western Australia, Western Australia, Australia.

${ }^{3}$ Water Corporation, Western Australia, Australia.

${ }^{4}$ Enviromental Health Directorate, Health Department of Western Australia, Western Australia, Australia.

${ }^{5}$ School of Biomedical Sciences, University of Western Australia, Crawley, Western Australia, Australia.

${ }^{6}$ School of Medicine, University of Western Australia, Crawley, Western Australia, Australia.

* Correspondence:

Avram.levy@ health.wa.gov.au

Keywords: SARS-CoV-2, genomic epidemiology, wastewater surveillance, public health microbiology, whole genome sequencing

Word count: 3400; Figures =1; Tables =1.

\begin{abstract}
After a limited first wave of community transmission in March 2020, Western Australia has remained largely free of COVID-19, with cases restricted to hotel quarantine, commercial vessels, and small, infrequent community clusters. Despite so few cases, whole genome sequencing (WGS) of SARS-CoV-2 from wastewater of large municipal treatments plants yielded genomic coverage up to 98\% with sufficient depth to link wastewater to the WGS sequences of active cases in the catchment at the time. WGS analysis of wastewater contemporaneous with clinical cases can also be used to rule out transmission between cases in different catchments, when their SARS-CoV-2 genomes differ. These findings reveal a greater potential of wastewater WGS to inform outbreak control and disease surveillance than previously recognized.
\end{abstract}




\section{Introduction}

Western Australia (WA) is geographically the largest state in Australia, with a total land area of approximately 2.5 million $\mathrm{km}^{2}$, representing a third of Australia's total land area. The state borders remote regions of South Australia and the Northern Territory as well as the Indian and Southern Oceans, creating a considerable buffer to undetected COVID-19 introductions by land. Almost $80 \%$ of the 2.67 million inhabitants reside in the capital city of Perth which is situated near the South West corner of WA. Perth is currently the only arrival point for commercial international flights into WA.

The first COVID-19 case in Western Australia (WA) was notified on the $21^{\text {st }}$ of February 2020, with case numbers rapidly increasing before widespread restrictions took effect. Strict regulations on movements, closure of non-essential businesses, closed state and international borders to nonresidents and mandatory supervised 14-day hotel quarantine of returning residents ended community transmission in WA. As of the $11^{\text {th }}$ of November 2021, 1,110 confirmed COVID-19 cases had been reported in WA with $89 \%$ of cases acquired in another country or while at sea, $1 \%$ acquired in another Australian jurisdiction, and the remaining 10\% of infections acquired from a known close contact or unknown source of infection within WA (1).

Samples from all COVID-19 cases in WA are referred for whole-genome sequencing (WGS) to determine SARS-CoV-2 lineages and Variants of Concern (VOCs). Genomic analyses are used to identify, confirm or disprove transmission clusters in hotel quarantine, in the community or other settings.

In patients with COVID-19, viral shedding in faeces is common. Persistence in stool even when SARS-CoV-2 can no longer be detected in respiratory samples has also been reported. Diarrhoea in COVID-19 patients has been described with varying incidences from $3.8 \%$ to $80 \%$ of cases with virus titres in stool also varying considerably $\left(10^{3}\right.$ to $10^{8}$ RNA copies/g of stool) (2-4). In addition to shedding via stool, wastewater captures nasopharyngeal secretions and urine, which may also contribute to SARS-CoV-2 RNA levels in wastewater $(4,5)$.

Early in the pandemic wastewater based epidemiology was implemented in several countries to track introduction and spread of SARS-CoV-2, primarily using RT-PCR (6-14). In Australia a network involving laboratories, wastewater (WW) utilities and public health units was established in 2020 to implement SARS-CoV-2 wastewater surveillance across the nation and integrate the results with health data ${ }^{1}$. With limited community transmission in Australia, the goal has been to use SARS-CoV2 signals from WW to provide early warning of the introduction of SARS-CoV-2 into catchments with no known clinical cases, to use sub-catchment sampling to direct public health resources and community messaging to the likely locations of cases, and to monitor for clearance of virus from catchments to support the easing of restrictions post-outbreak. In WA, weekly monitoring of SARSCoV-2 at five metropolitan WW treatment plants commenced in November 2020, and then expanded early in 2021 to include a total of eighteen treatment plants spanning all geopolitical subdivisions with results subsequently made publicly available each week. Only one catchment regularly returned positive results, the catchment encompassing quarantine hotels where active COVID-19 cases are isolated. Targeted sub-catchment sampling is also initiated on an ad hoc basis in response to small community clusters.

\footnotetext{
${ }^{1}$ https://www.waterra.com.au/project-details/264
} 
The use of WGS for SARS-CoV-2 wastewater surveillance has been described in regions with considerable SARS-CoV-2 community transmission. Sequences from wastewater have been compared to lineages known to be circulating in the community at the time (15-18). Wastewater genomics has been used to detect single nucleotide polymorphisms (SNPs) and low frequency variants not reported in clinical cases residing within the corresponding region in order to provide an early warning of emerging new variants (16-19). Where clinical testing is minimal, wastewater SARS-CoV-2 WGS has identified the proliferation of VOC sequences in communities (15).

In areas with low COVID-19 case numbers and limited community transmission, SARS-CoV-2 RNA copies in wastewater are typically very low and towards the limit of detection for real-time RT-PCR, making them unsuitable for WGS. However, recent experience in WA demonstrates that sufficient RNA can be amplified from catchments exceeding 700,000 population despite less than five active cases shedding into the wastewater. Sequences covering the majority of the SARS-CoV-2 genome can be assembled and linked to individuals if their SARS-CoV-2 genomes are known and have sufficient diversity to be differentiated from each other. In this study we show sufficient resolution of wastewater procured genomes can be obtained from a community with few COVID-19 cases thereby contributing to the epidemiological investigation by providing geospatial SARS-CoV-2 strain information.

\section{Methods}

In Western Australia all samples from clinical COVID-19 cases are submitted for WGS as part of the public health response, unless viral load is deemed insufficient (generally $\mathrm{Ct}$ above 36.0). Wastewater sampling also occurs as part of the public health response to the COVID-19 pandemic with five municipal treatment plants ranging in catchment size from approximately 90,000 to 740,000 sampled weekly from April 2020. In 2021 the program was expanded to include a total of eighteen metropolitan and regional sites. Composite samples were collected using ISCO autosampler devices, flow paced and continual with 400mL sampled each hour from six metropolitan wastewater treatment plants. $250 \mathrm{~mL}$ aliquots were collected and shipped to the laboratory on ice, arriving on the same day and tested within 24 hours. All quarantine hotels are located in the Perth $\mathrm{CBD}$ and contribute to a wastewater treatment plant catchment with total population size of approximately 250,000 .

$50 \mathrm{~mL}$ wastewater samples were pre-treated to achieve a final $\mathrm{MgCl}_{2}$ concentration of $25 \mathrm{mM}$ and centrifuged to separate solids at $4,600 \mathrm{rpm}$ for 30 minutes at $4{ }^{\circ} \mathrm{C}$. The supernatant was concentrated via vacuum filtration of $50 \mathrm{~mL}$ onto $0.45 \mu \mathrm{M}$ electronegative filter membrane in a Microcheck® beverage monitor (Pall, United States), as described previously (20). Each filter membrane was added to $800 \mathrm{uL}$ of lysis buffer in a MagMAX ${ }^{\mathrm{TM}}$ Microbiome Bead Tube (ThermoFisher, United States) and homogenised on a TissueLyser II (Qiagen, Germany) for 10 minutes at 10,000rpm then centrifuged at 14,000rpm for 2 minutes. RNA was purified from 400uL aliquots using the $\operatorname{MagMax}^{\mathrm{TM}}$ Microbiome Ultra Nucleic Acid Isolation Kit (ThermoFisher, United States) on the MagMAX ${ }^{\mathrm{TM}}$ Express-96 (ThermoFisher, United States) according to manufacturer's protocol. MS2 bacteriophage was added at a known concentration to the sample plate as an extraction and amplification control. Final nucleic acid elution occurred in $100 \mu \mathrm{L}$ of supplied elution buffer. Isolated nucleic acid was assayed immediately on the PerkinElmer® SARS-CoV-2 Real-time RTPCR assay (PerkinElmer, United States) in two technical replicates using half volume of the 
mastermix. Nucleic acid was diluted four-fold to minimise PCR inhibition (10 $\mu \mathrm{L}$ Mastermix, $15 \mu \mathrm{L}$ Ultra-Pure $\mathrm{H}_{2} \mathrm{O}, 5 \mu \mathrm{L}$ nucleic acid).

For WGS, cDNA synthesis occurred immediately upon completion of screening PCR using SuperScript ${ }^{\mathrm{TM}}$ VILO $^{\mathrm{TM}}$ mastermix (ThermoFisher, United States). Tiled amplicons covering the full genome of SARS-CoV-2 were amplified using both a modified ARTIC V2 (1000bp) primer set and the ARTIC V3 (400bp) primer set in separate reactions in conjunction with Q5 Hot Start DNA Polymerase (New England BioLabs, United States) as per recommended protocol ${ }^{2}$. Each wastewater sample comprised of two to four technical replicates of the ARTIC V2 and V3 amplicons.

Illumina Nextera XT (Illumina, United States) libraries were created as per manufacturer recommendations, but using half volume of reagents and unique dual indexing. Sequencing was by Illumina iSeq (Illumina, United States). Run QC and sequence analysis was performed by custom inhouse pipeline and Geneious R11 (Biomatters, New Zealand). Fastq files from the replicates were analysed independently and also as merged BAM files. COVID-19 cases in hotel quarantine and in the community were identified by review of WA Health Department records. Collection dates, RTqPCR Ct values, consensus whole genome sequences and Pangolin lineages of clinical cases were all obtained from PathWest records. Primer and probe sequences of the Perkin Elmer kit were identified as the China CDC set (21) and obtained from (22).

Sequences were aligned using MAFFT v7.467, and then manually corrected in Geneious Prime. Western Australian SARS-CoV-2 genome sequences were assigned to lineages using the Phylogenetic Assignment of Named Global Outbreak LINeages (PANGOLIN) tool ${ }^{3}$. The bioinformatics analysis was performed by identifying and comparing SNP profiles of wastewater sequences to known active cases, defined as the date of specimen collection of the first positive test, to four weeks after the case was clinically cleared. Sites at which there were SNPs to the Wuhan-Hu1 sequence (NC_045512.2) were identified for both the wastewater and for all active cases in the catchment at the time.

Separate 50mL aliquots of SARS-CoV2 PCR positive wastewater samples were also concentrated on electronegative membranes and each filter membrane was added to 800uL of Minimal Essential Media with 2\% Foetal bovine serum in a MagMAX ${ }^{\mathrm{TM}}$ Microbiome Bead Tube (ThermoFisher, United States). These were homogenised on a TissueLyser (Qiagen, Germany) for 10 minutes at 10,000rpm and centrifuged at 14,000rpm for 2 minutes. The culture media was separated and diluted to achieve a total volume of $1 \mathrm{~mL}$, then inoculated onto Vero E6 cells for SARS-CoV-2 culture. At 7 days incubation, TCF's from the initial cultures were passaged onto fresh cells. Passage was repeated twice and cells were monitored for cytopathic effect.

\section{Results}

In May and June 2021 three WW samples from the quarantine hotel catchment in Perth produced lower SARS-CoV-2 RT-sqPCR Ct values than typically seen, with the first two samples also negative for the $\mathrm{N}$ gene target (ORF1ab positive only). There were few active cases in the catchment throughout the time of sampling. Sequencing of all three WW samples produced reads covering

${ }^{2}$ https://www.nebiolabs.com.au/protocols/2019/07/02/pcr-using-q5u-hot-start-high-fidelity-dna-polymerase-neb-m0515

${ }^{3}$ https://github.com/cov-lineages/pangolin 
$>90 \%$ of the reference genome with variable depth (Table 1; samples A-C). The first two WW samples failed to react in the $\mathrm{N}$ gene target of the PerkinElmer SARS-CoV-2 screening assay despite a stronger than usual $\mathrm{Ct}$ in the ORF1ab target. Comparison of the China CDC primer and probe binding regions $(21,22)$ with the WW composite genomes identified a 3 bp substitution GGG to AAC spanning across 2 amino acids causing mutations N:R202K and N:G203R at the 5 ' end of the China CDC forward primer-binding region of the $\mathrm{N}$ gene (Figure 1). A 3bp (del:28896:3) deletion was also identified in the 3' end of the primer binding region of the same primer (Figure 1 inset). The substitution and deletion were identified in $100 \%$ of combined WW reads covering that genomic site and subsequently identified in GISAID and WA sequences of lineage B.1.1.318, including those from cases in hotel quarantine at the time of the first two WW samples. Subsequent testing of the WA B.1.318 lineage samples $(n=10)$ by the RT-sqPCR kit used for wastewater confirmed the $\mathrm{N}$ target drop-out occurred in a concentration-dependent manner.

Of the active cases at the time of the first WW sample (Table 1; sample A), only one case was assigned the B.1.1.318 lineage. A SNP comparison showed three SNPs from the WW sample were found in all active cases and all 29 SNPs identified in the WW sequence were found only in the case assigned B.1.1.318. All remaining active cases shared 4-10 SNPs with the WW sample. Although we could not rule out partial contributions by the other remaining active cases, we were able to determine that the B.1.1.318 case was contributing to the positive result. This B.1.1.318 active case had an unusual pattern of respiratory sample PCR positivity, with Cts in the range $24.5-41.8$ for approximately three weeks.

Two weeks later a second WW sample with the same pattern of $\mathrm{N}$ gene drop-out also produced a WGS with good coverage and depth (Table 1; sample B). This sample coincided with a subsequent B.1.1.318 case in hotel quarantine. This later case could be differentiated from the first by 17 distinct SNPs. The wastewater sample again predominantly matched the B.1.1.318 active in the catchment at the time, rather than the other active cases which were of other lineages. The prior B.1.1.318 case may have still been shedding virus into the catchment but none of the 17 distinct SNPs evident in the consensus sequence of prior case were apparent in the second WW sample.

One week later a third WW sample with unusually strong Cts in both ORF1ab and $\mathrm{N}$ gene occurred in the same catchment service quarantine hotels (Table 1; sample C). By this time there was a cluster of unrelated community cases in a separate catchment of WW from suburbs to the north of Perth. Several cases in hotel quarantine and the northern suburbs cluster were assigned the B.1.617.2 lineage (Delta variant), but could be distinguished from each other by a number of SNPs. This third WW sample had a total of 31 SNPs to the reference sequence and was highly similar (30 and 29 SNPs) to two cases in hotel quarantine, a family cluster that had recorded extremely strong Cts (as low as 13) from respiratory samples at the time of the WW collection. Seven SNPs present in the WW sample were found exclusively in the two cases and not in the northern suburbs cluster.

Sub catchment sampling was initiated in response to the northern suburbs cluster, with one sub catchment of population approximately 100,000 within the very large northern suburb catchment $(\sim 700,000)$ being positive, consistent with the location of the known positive cases quarantining in their homes. A WW sample from this sub catchment produced WGS sequence with coverage of $93.6 \%$ but at a relatively low depth of 588x. Comparison of this WW sample to sequences from the cluster active at the time showed only one heterozygous nucleotide location where the alternate base identified in $60 \%$ of reads (G13332C) could not be accounted for by the cluster sequences.

None of the wastewater samples produced observable cytopathic effect with cell culture. 


\section{Discussion}

This wastewater genomic epidemiology study provides several examples where it was possible to trace back and link composite SARS-CoV-2 genomes obtained from WW to individuals in hotel quarantine. Furthermore, during an episode of limited community transmission, a comparative analysis of SNP profiles in the composite genome from WW with the contemporaneous clinical sample genomic sequences ruled out the cases in hotel quarantine being the source of the community transmission observed in a separate catchment. The source of the community transmission was subsequently identified to be a returned traveller from another Australian jurisdiction. The very small number of COVID-19 cases in WA (1) along with a rigorous WGS approach has created opportunities to link detections in wastewater to corresponding known clinical cases isolating in hotels.

Due to the low prevalence of SARS-CoV-2 in WA post-implementation of WW surveillance, only the treatment plant servicing the quarantine hotels regularly returned positive RT-qPCR results. Detections of SARS-CoV-2 in WW were always weak despite the highly sensitive PCR assay used, with Cts from auto-samplers at treatment plants not less than 36 cycles. The observation of stronger than usual Cts in May and July was therefore unprecedented in our experience, with several factors potentially contributing. Cooler ambient wastewater temperatures coinciding with the seasonal drop in temperature in Perth may have improved persistence of RNA in the WW systems. Temperature has been shown to affect SARS-CoV-2 RNA detections in spiking experiments (23).

Two of the three situations where near complete composite genomes were obtained involved cases of B.1.1.318 lineage. This lineage is uncommon in Australian sequences submitted to the GISAID repository (24), but had been circulating globally, peaking in April to May 2021 (CoV-Lineages ${ }^{4}$ ) and responsible for a large proportion of cases in some regions (25). Known cases of lineage B.1.1.318 in WA were reviewed and were found to coincide with a slight drop in WW Cts at the time. It is plausible that there are virological determinants of viral shedding with higher viral load in stool associated with infections by particular lineages, however there is no published evidence to support this. The two cases of lineage B.1.1.318 which led to the stronger than usual WW signals were both partially vaccinated. It is unknown if the faecal shedding of SARS-CoV-2 is altered in post-vaccination infection.

SARS-CoV-2 RNA in WW is widely regarded as present in viral fragments and there are no reports of virus culture from wastewater despite documented efforts $(13,26)$. Our attempts were similarly unsuccessful, although the method was not developed or optimized for SARS-CoV-2 in WW. The ability to distinguish between inactive but intact virion and fragmented virus has been described (13, 23) but was not attempted in this study. It is possible that the high genomic coverage results, as well as the greater than expected PCR signal strengths suggest intact virion may have been present in the WW samples. However, this theory was not specifically assessed.

Highly sensitive detection and genomic characterisation of SARS-CoV-2 relies on PCR amplification which introduces an intrinsic bias towards higher copy number template in the specimen. RNA from cases weakly shedding virus into the WW is likely to be dominated by RNA from cases with a higher copy number when creating composite genomes. Therefore sequence diversity in composite genomes

\footnotetext{
${ }^{4}$ https://cov-lineages.org/lineage.html?lineage=B.1.1.318
} 
from WW is likely to underestimate the true sequence diversity in the WW, instead representing the most common fragments only. The use of multiple grab samples alongside 24 hour collections by auto or passive sampling devices in certain settings may provide additional sequence as it may increase the representation from individuals over different time points.

For two of the wastewater samples in this study, single contributing cases were identified as the most likely sources and in both instances the cases were infected with SARS-CoV-2 of lineage B.1.1.318. ORF1ab targets of the commercial SARS-CoV-2 PCR either reacted poorly or were negative with WW. WGS results identified a deletion in the forward primer binding region, which was also present in a large proportion of GISAID sequences of lineage B.1.1.318 (24). Previous WA sequences of B.1.1.318 had the same deletion and when the samples were retrieved and tested by the WW PCR assay showed a concentration dependent signal delay and drop-out of the ORF1ab target.

Although each WW sample could be linked to specific samples through multiple unique SNPs, there was one heterozygous base identified in the WW results which did not correspond to any known active or historical cases. A broader search for sequences with the alternate base at that location perform on the GISAID and NCBI database also did not yield any results, suggesting that the SNPs from the WW samples were template misreads introduced during the PCR stage.

Wastewater-based epidemiology has become a valuable tool for screening large populations for the presence of SARS-CoV-2 viral fragments whilst individuals within the population remain anonymous. This study demonstrates the previously unrecognised ability of WW genomic surveillance to identify the individual contributors to positive WW samples by comparing the results to clinical sequence data. This is only possible in the uncommon situation where communities are free of more widespread COVID-19, and the location of all active cases in regards to WW catchment are likely known and have their genomic sequences from respiratory samples available for comparison. In communities with multiple contributors of SARS-CoV-2 to WW, all with very similar or identical genomes contributing to composite WW genomes, it has not been possible to understand the discriminatory power of genomic methods targeting WW RNA (15-19). This study demonstrates that only a few contributing cases are necessary to provide near-complete genomes, even in catchments of up to a quarter of a million people. The events leading to why some individuals contribute more to the composite sequences in these situations remains poorly understood and warrants further work.

The application of sensitive genomic approaches in combination with microbial surveillance data has the potential to provide early warnings of the presence of new and emerging pathogen variants, as well as their persistence and eradication. As a result of COVID-19, the scope of WW microbial surveillance has evolved from focussing on gastrointestinal viruses to informing public health responses to an evolving respiratory pathogen. The introduction of genomics creates a much more powerful tool for interrogation of PCR detections from WW. These technologies are likely to be applicable to an expanded range of pathogens of public health significance as well as for monitoring of antimicrobial resistance.

\section{Acknowledgements}

We appreciate the advice on cell culture and virus isolation methods provided by Dr Chisha Sikazwe and thank the WGS scientists and COVID wastewater team at PathWest. 
Figure 1: Alignment of GISAID B.1.1.318 sequences compared to Wuhan reference showing 3bp substitution (circled in green) and 3 bp deletion (circled in blue). Inset shows WW sample reads mapped to Wuhan reference and China CDC N gene forward primer.

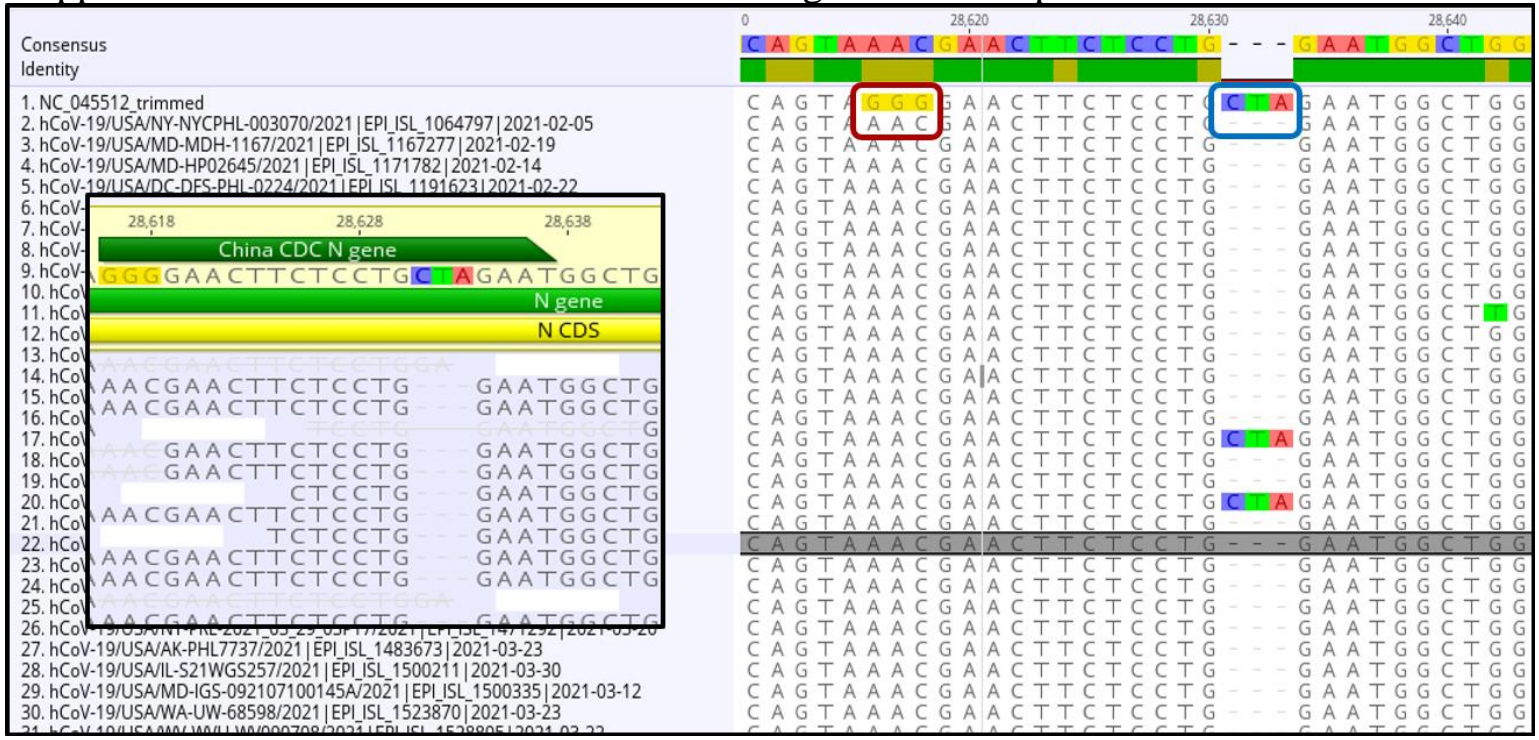

Table 1: Wastewater sample descriptions, SARS-CoV-2 PCR and WGS results

\begin{tabular}{|c|c|c|c|c|c|c|c|c|c|c|}
\hline Sample & Catchment & $\begin{array}{c}\text { Date } \\
\text { collected }\end{array}$ & $\begin{array}{l}\text { Catchment } \\
\text { size }\end{array}$ & $\begin{array}{l}\text { Number } \\
\text { of active } \\
\text { cases in } \\
\text { catchment }\end{array}$ & $\begin{array}{c}\mathrm{PE} \mathrm{N} \\
\text { gene } \\
\mathrm{Ct}^{*}\end{array}$ & $\begin{array}{c}\text { PE } \\
\text { ORF1ab } \\
\mathrm{Ct}^{*}\end{array}$ & $\begin{array}{c}\text { Total } \\
\text { reads** }\end{array}$ & $\% \mathrm{GC}^{* *}$ & $\begin{array}{c}\text { Genome } \\
\text { coverage** }\end{array}$ & $\begin{array}{l}\text { Average } \\
\text { depth** }\end{array}$ \\
\hline A & $1 * * *$ & $25 / 05 / 2021$ & 247,777 & 3 & ND & 36.5 & $1,012,339$ & 39.1 & 99.7 & 4095 \\
\hline B & 1 & $15 / 06 / 2021$ & 247,777 & 2 & ND & 36.5 & 188,212 & 39.3 & 92.0 & 763 \\
\hline $\mathrm{C}$ & 1 & $29 / 06 / 2021$ & 247,777 & 5 & 35.9 & 36.5 & 993278 & 39.4 & 95.7 & 4161 \\
\hline D & 2 & $29 / 06 / 2021$ & 87,483 & 1 & 38.7 & 39.2 & 66178 & 39.9 & 54.2 & 279 \\
\hline $\mathrm{E}$ & 1 & $1 / 07 / 2021$ & 247,777 & 2 & 38.0 & 38.3 & 203928 & 39.7 & 90.5 & 868.1 \\
\hline $\mathrm{F}$ & 3 & $2 / 07 / 2021$ & 698,299 & 3 & 37.60 & 39.7 & 1220 & 39.1 & 4.9 & 4.8 \\
\hline G & $3 f$ & $3 / 07 / 2021$ & 99,191 & 2 & 36.5 & 37.8 & 141655 & 38.9 & 93.6 & 588.7 \\
\hline $\mathrm{H}$ & 1 & $7 / 07 / 2021$ & 247,777 & 5 & 37.8 & 38.8 & 4 & 35.4 & 0 & 0.01 \\
\hline I & 2 & $8 / 07 / 2021$ & 87,483 & 2 & 38.7 & ND & 1803 & 40.3 & 71.2 & 8 \\
\hline
\end{tabular}

*Average of two replicates; **Sum of replicates; *** Catchment 1 is the hotel quarantine catchment; $\mathrm{ND}=$ not detected. 


\section{References}

1. Spatial Services Unit EB, Public and Aboriginal Health Division, Department of Health, Government of Western Australia;. Coronavirus COVID-19 in Western Australia https://experience.arcgis.com/experience/359bca83a1264e3fb8d3b6f0a028d768 [Accessed October 11, 2021] [Updated October 11, 2021].

2. Foladori P, Cutrupi F, Segata N, Manara S, Pinto F, Malpei F, et al. SARS-CoV-2 from faeces to wastewater treatment: What do we know? A review. Sci Total Environ. 2020;743:140444. doi: 10.1016/j.scitotenv.2020.140444

3. Kitajima M, Ahmed W, Bibby K, Carducci A, Gerba CP, Hamilton KA, et al. SARS-CoV-2 in wastewater: State of the knowledge and research needs. Sci Total Environ. 2020;739:139076. doi: 10.1016/j.scitotenv.2020.139076

4. Jones DL, Baluja MQ, Graham DW, Corbishley A, McDonald JE, Malham SK, et al. Shedding of SARS-CoV-2 in feces and urine and its potential role in person-to-person transmission and the environment-based spread of COVID-19. Sci Total Environ. 2020;749:141364. doi: 10.1016/j.scitotenv.2020.141364

5. Peng L, Liu J, Xu W, Luo Q, Chen D, Lei Z, et al. SARS-CoV-2 can be detected in urine, blood, anal swabs, and oropharyngeal swabs specimens. J Med Virol. 2020;92(9):1676-80. doi: 10.1002/jmv.25936

6. Ahmed W, Angel N, Edson J, Bibby K, Bivins A, O'Brien JW, et al. First confirmed detection of SARS-CoV-2 in untreated wastewater in Australia: A proof of concept for the wastewater surveillance of COVID-19 in the community. Sci Total Environ. 2020;728:138764. doi: 10.1016/j.scitotenv.2020.138764

7. Chavarria-Miró G, Anfruns-Estrada E, Martínez-Velázquez A, Vázquez-Portero M, Guix S, Paraira M, et al. Time Evolution of Severe Acute Respiratory Syndrome Coronavirus 2 (SARS-CoV2) in Wastewater during the First Pandemic Wave of COVID-19 in the Metropolitan Area of Barcelona, Spain. Appl Environ Microbiol. 2021;87(7). doi: 10.1128/aem.02750-20

8. La Rosa G, Iaconelli M, Mancini P, Bonanno Ferraro G, Veneri C, Bonadonna L, et al. First detection of SARS-CoV-2 in untreated wastewaters in Italy. Sci Total Environ. 2020;736:139652. doi: 10.1016/j.scitotenv.2020.139652

9. Medema G, Been F, Heijnen L, Petterson S. Implementation of environmental surveillance for SARS-CoV-2 virus to support public health decisions: Opportunities and challenges. Curr Opin Environ Sci Health. 2020;17:49-71. doi: 10.1016/j.coesh.2020.09.006

10. Nemudryi A, Nemudraia A, Wiegand T, Surya K, Buyukyoruk M, Cicha C, et al. Temporal Detection and Phylogenetic Assessment of SARS-CoV-2 in Municipal Wastewater. Cell Rep. 2020;1(6):100098. doi: 10.1016/j.xcrm.2020.100098

11. Randazzo W, Truchado P, Cuevas-Ferrando E, Simón P, Allende A, Sánchez G. SARS-CoV2 RNA in wastewater anticipated COVID-19 occurrence in a low prevalence area. Water Res. 2020;181:115942. doi: 10.1016/j.watres.2020.115942

12. Wu F, Zhang J, Xiao A, Gu X, Lee WL, Armas F, et al. SARS-CoV-2 Titers in Wastewater Are Higher than Expected from Clinically Confirmed Cases. mSystems. 2020;5(4). doi:

10.1128/mSystems.00614-20 
13. Rimoldi SG, Stefani F, Gigantiello A, Polesello S, Comandatore F, Mileto D, et al. Presence and infectivity of SARS-CoV-2 virus in wastewaters and rivers. Sci Total Environ. 2020;744:140911. doi: 10.1016/j.scitotenv.2020.140911

14. Wurtzer S, Marechal V, Mouchel J, Maday Y, Teyssou R, Richard E, et al. Evaluation of lockdown effect on SARS-CoV-2 dynamics through viral genome quantification in waste water, Greater Paris, France, 5 March to 23 April 2020. Euro Surveill. 2020;25(50):2000776. doi: 10.2807/1560-7917.ES.2020.25.50.2000776

15. Bar-Or I, Weil M, Indenbaum V, Bucris E, Bar-Ilan D, Elul M, et al. Detection of SARSCoV-2 variants by genomic analysis of wastewater samples in Israel. Sci Total Environ. 2021;789:148002. doi: 10.1016/j.scitotenv.2021.148002

16. Crits-Christoph A, Kantor RS, Olm MR, Whitney ON, Al-Shayeb B, Lou YC, et al. Genome Sequencing of Sewage Detects Regionally Prevalent SARS-CoV-2 Variants. mBio. 2021;12(1):e02703-20. doi: 10.1128/mBio.02703-20

17. Izquierdo-Lara R, Elsinga G, Heijnen L, Munnink BBO, Schapendonk CME, Nieuwenhuijse D, et al. Monitoring SARS-CoV-2 Circulation and Diversity through Community Wastewater Sequencing, the Netherlands and Belgium. Emerg Infect Dis. 2021;27(5):1405-15. doi: 10.3201/eid2705.204410

18. Fontenele RS, Kraberger S, Hadfield J, Driver EM, Bowes D, Holland LA, et al. Highthroughput sequencing of SARS-CoV-2 in wastewater provides insights into circulating variants. Water Res. 2021;205:117710. doi: 10.1016/j.watres.2021.117710

19. Jahn K, Dreifuss D, Topolsky I, Kull A, Ganesanandamoorthy P, Fernandez-Cassi X, et al. Detection and surveillance of SARS-CoV-2 genomic variants in wastewater. medRxiv. 2021:2021.01.08.21249379. doi: 10.1101/2021.01.08.21249379

20. Ahmed W, Bertsch PM, Bivins A, Bibby K, Farkas K, Gathercole A, et al. Comparison of virus concentration methods for the RT-qPCR-based recovery of murine hepatitis virus, a surrogate for SARS-CoV-2 from untreated wastewater. Sci Total Environ. 2020;739:139960. doi: 10.1016/j.scitotenv.2020.139960

21. Water Research Australia. Project 2064 - Method evaluation and optimisation: Investigation of PCR-based methods and feasibility study for whole-genome sequencing. Jex Laboratory, The Walter and Eliza Hall Institute of Medical Research Subproject: Molecular tools for the detection and verification of SARS-CoV-2 in wastewater. 2021. https://www.waterra.com.au/project-details/276

22. Suo T, Liu X, Feng J, Guo M, Hu W, Guo D, et al. ddPCR: a more accurate tool for SARSCoV-2 detection in low viral load specimens. Emerg Microbes Infect. 2020;9(1):1259-68. doi: $10.1080 / 22221751.2020 .1772678$

23. Wurtzer S, Waldman P, Ferrier-Rembert A, Frenois-Veyrat G, Mouchel JM, Boni M, et al. Several forms of SARS-CoV-2 RNA can be detected in wastewaters: Implication for wastewaterbased epidemiology and risk assessment. Water Res. 2021;198:117183. doi: 10.1016/j.watres.2021.117183

24. Elbe S, Buckland-Merrett G. Data, disease and diplomacy: GISAID's innovative contribution to global health. Glob Chall. 2017;1(1):33-46. doi: 10.1002/gch2.1018

25. Tegally H, Ramuth M, Amoaka D, Scheepers C, Wilkinson E, Giovanetti M, et al. Genomic epidemiology of SARS-CoV-2 in Mauritius reveals a new wave of infections dominated by the 
B.1.1.318, a variant under investigation. medRxiv. 2021:2021.06.16.21259017. doi:

10.1101/2021.06.16.21259017

26. Wang J, Feng H, Zhang S, Ni Z, Ni L, Chen Y, et al. SARS-CoV-2 RNA detection of hospital isolation wards hygiene monitoring during the Coronavirus Disease 2019 outbreak in a Chinese hospital. Int J Infect Dis. 2020;94:103-6. doi: 10.1016/j.ijid.2020.04.024 